\title{
Tracheal melanic tattoo
}

\author{
M. Mondoni ${ }^{1}$, P. Carlucci ${ }^{1}$, L. Boiocchi², S. Centanni ${ }^{1}$
}

A 70-years old white female was referred to our Pulmonary Department after undergoing a chest X-ray which revealed two bilateral lung masses.

Her past medical history included diverticulosis of colon and hypothyroidism; five years earlier the patient had also undergone an excisional biopsy of a central back melanoma (Clark level III), and yearly follow-up chest Xrays were performed.

She was a non smoker and denied any alcohol intake.

On admission she was asymptomatic and a physical examination did not reveal any abnormalities. Her only medication was $75 \mathrm{mcg}$ of daily oral levotiroxine.

Routine hematologic blood tests were unremarkable and blood gases were within normal limits.

The patient underwent a chest CT scan that revealed two round lung masses located in the lower lobes (fig. 1); there was no evidence of hilar or mediastinal enlarged lymph nodes.

The PET-CT scans showed a pathological FDG uptake at the level of the lung lesions with SUV max of 11.1 and 13, very suggestive of malignancy. There were no other body areas of increased tracer activity.

A bronchoscopy only revealed a centimetric flat and black pigmented area in the pars membranacea of the trachea (fig. 2). Suspecting a tracheal localisation of melanoma, several biopsies were performed.

The pathological analysis demonstrated fragments of respiratory mucosa and connective tissue with numerous medium sized cells with a granular cytoplasm filled with brown pigment. Immunohistochemistry stains were negative for HMB45 and S100, but intensely positive to CD68, revealing their hystiocytic nature.

A transthoracic CT-guided needle aspiration of a lung mass was then performed and histological examination revealed lung melanoma metastasis. The paraffin embedded biopsy fragments were composed by a population of round and fusiform atypical cells intensely stained with immunohistochemistry for HMB45 and S100, confirming their melanocytic derivation (fig. 3).

The patient was treated with 4 cycles of intravenous dacarbazine and after 3 months of

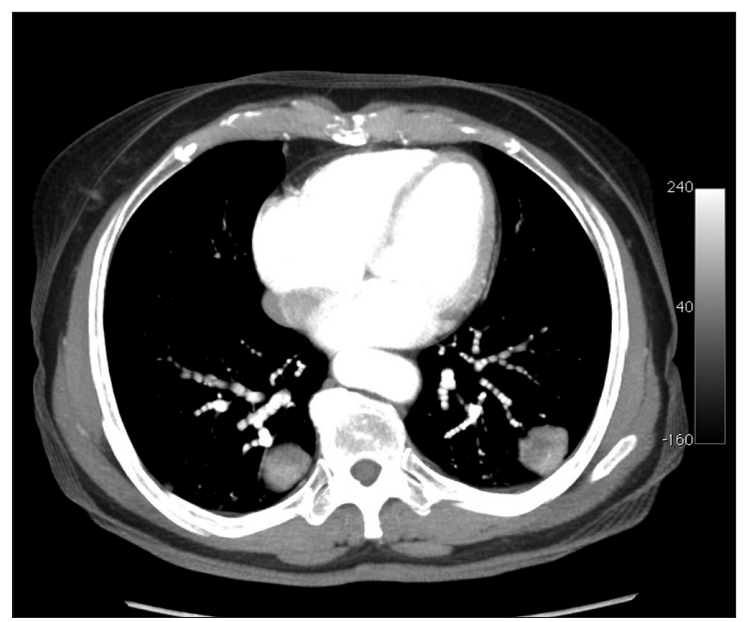

Fig. 1. - Chest CT scan revealing two round lung masses in the lower lobes.

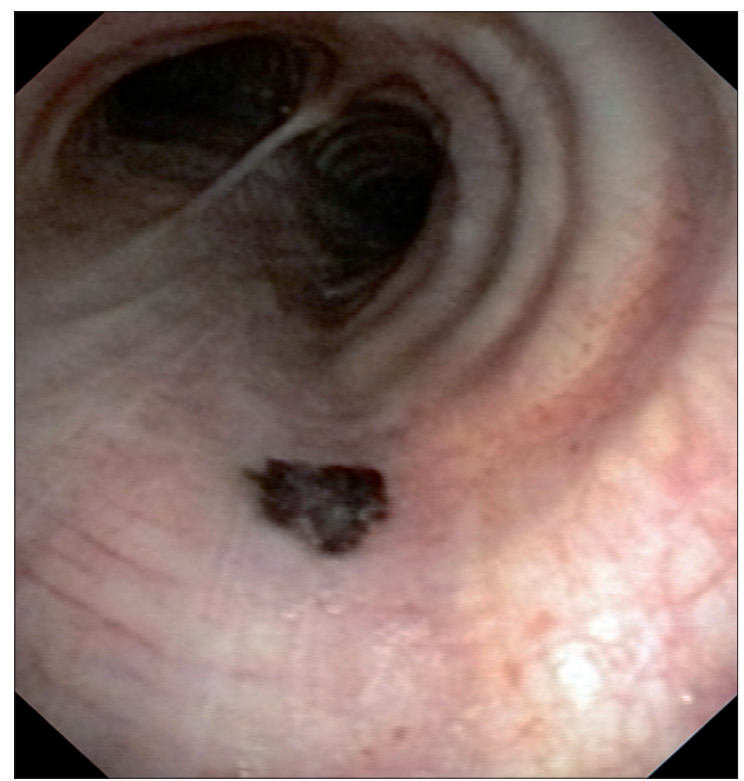

Fig. 2. - Centimetric flat and black pigmented area in the pars membranacea of the trachea.
Monaldi Arch Chest Dis 2011; 75: 2, 147-148

Keywords:

Bronchoscopy,

Melanoma, Histology.

1 Pulmonary Division,

San Paolo Hospital,

University of Milan

2 Pathology Unit,

University of Milan,

IRCCS Ca' Granda

- Ospedale Maggiore

Policlinico Foundation,

Milan, Italy.

Correspondence:

Michele Mondoni, MD,

Pulmonary Division,

San Paolo Hospital,

University of Milan,

Via A. di Rudinì 8,

20142, Milan, Italy;

e-mail: michele.mondoni

@ao-sanpaolo.it 


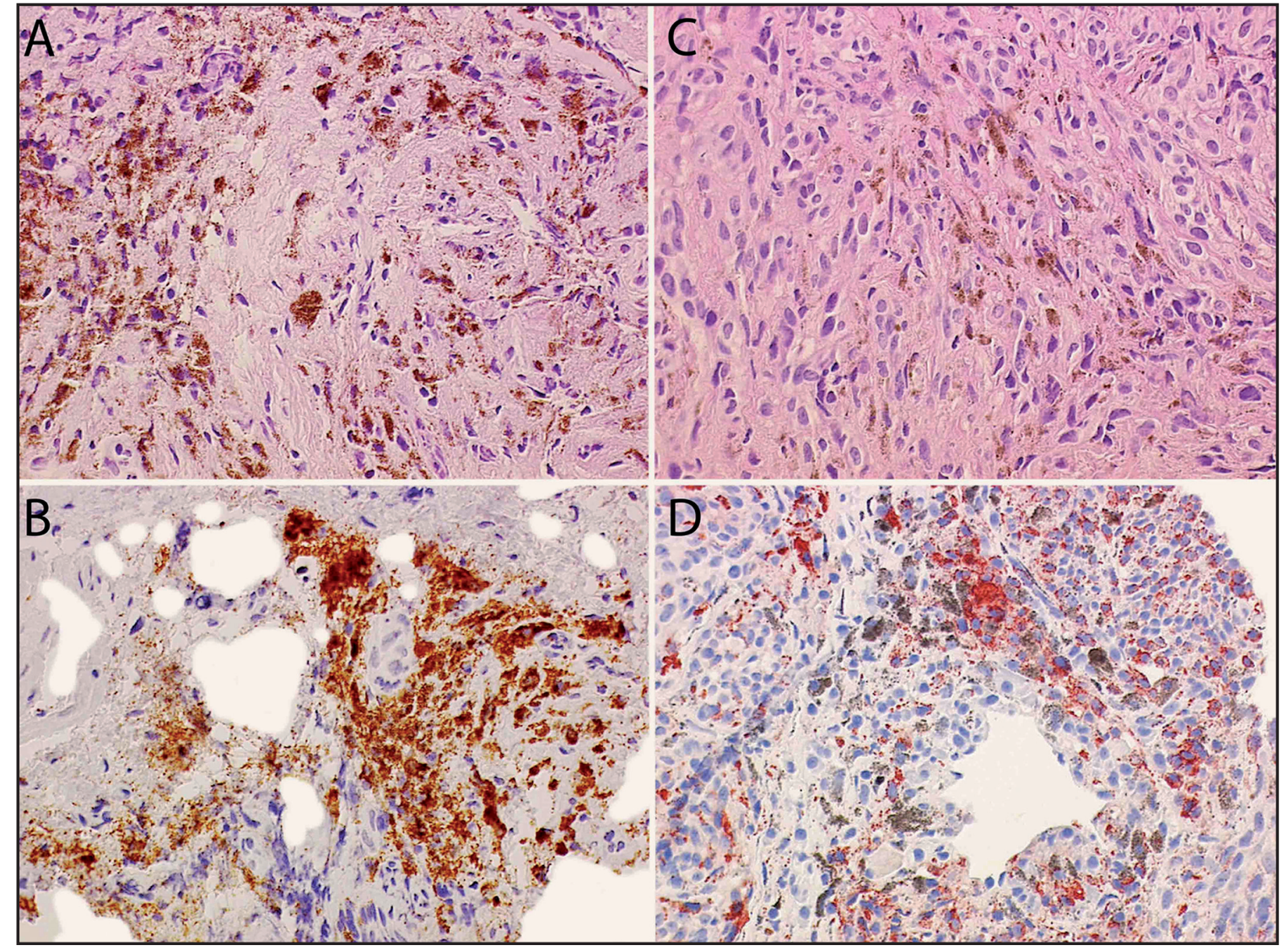

treatment she underwent a PET-CT scans that evidenced a disease progression with an increase in dimension and FDG uptake of the lung masses.

The patient, still asymptomatic, then started treatment with fotemustine but after 1 month was lost at follow-up.

The lung is a frequent site of metastases from malignant melanoma [1].

Very few cases of primary tracheal melanoma have been described and specific criteria were developed to define this diagnosis $[2,3]$. However, the presence of even benign melanocytes in the bronchopulmonary system is not usual [4]. Nevertheless, the presence of melanin in tracheal histiocytes has never been described before.

Since it is difficult to provide an exhaustive explanation for this tracheal melanic tattoo, we hypothised a vascular or lymphatic drainage of melanin and a localised tracheal deposition of melanic pigment from the metastatic lung masses.

\section{References}

1. Gromet MA, Ominsky SH, Epstein WL, Blois MS. The thorax as the initial site for systemic relapse in malignant melanoma: a prospective survey of 324 patients. Cancer 1979; 44: 776-84.

2. Terra RM, Minamoto H, Junqueira JJ, Falzoni R, Pego-Fernandes PM, Biscegli Jatene F. Tracheal malignant melanoma: successful outcome with tracheal resection. Ann Thorac Surg 2008; 86: 308-10.

3. Barzò P, Minik K, Tuka P, Kiss JI. The clinical significance of the primary malignant melanoma of the lower respiratory tract and/or lung based on the analysis of published case reports and of two patients. Diagn Ther Endosc 1995; 1: 169-175.

4. Jennings TA, Axiotis CA, Kress Y, Carter D. Primary malignant melanoma of the lower respiratory tract. Report of a case and literature review. Am J Clin Pathol 1990; 94: 649-55.
Fig. 3. - Haematoxylin-eosin stained sections of the tattooed area of the trachea (A) and of the pulmonary mass (C), both showing a marked deposition of melanic pigment. CD68 immunostaining of the tracheal tattoo (B) showing that cells filled with melanic pigment are histiocytes. HMB45 immunostaining of the pulmonary mass (D) showing the presence of malignant melanocytes. (Original magnification 20x). 